\title{
Frontières
}

\section{Comprendre et agir sur le suicide chez les Premières Nations Quelques lunes après l'initiation}

\section{Michel Tousignant, Arlene Laliberté, Gilles Bibeau et Denise Noël}

Volume 21, numéro 1, automne 2008

Prévenir le suicide

URI : https://id.erudit.org/iderudit/037881ar

DOI : https://doi.org/10.7202/037881ar

Aller au sommaire du numéro

\section{Éditeur(s)}

Université du Québec à Montréal

ISSN

1180-3479 (imprimé)

1916-0976 (numérique)

Découvrir la revue

Citer cet article

Tousignant, M., Laliberté, A., Bibeau, G. \& Noël, D. (2008). Comprendre et agir sur le suicide chez les Premières Nations : quelques lunes après l'initiation.

Frontières, 21(1), 113-119. https://doi.org/10.7202/037881ar
Résumé de l'article

Depuis trois ans, une équipe multidisciplinaire s'est engagée à réfléchir et à développer des stratégies sur la prévention du suicide en milieu autochtone dans le cadre d'un vaste programme de recherche. Une telle entreprise pose des défis inusités à toutes les étapes de son développement. Dans cet article, l'équipe de recherche indique les difficultés rencontrées et les pistes de solution élaborées. La première partie décrit la dimension épidémiologique du suicide en milieu autochtone et elle présente les premiers résultats. La deuxième partie décrit le volet prévention et fait un bilan des essais entrepris. La troisième partie résume une recherche auprès des familles visant à mieux comprendre le contexte de socialisation dans trois milieux contrastés. Suit une brève description de l'objectif de formation de jeunes chercheurs autochtones. La discussion porte sur la capacité à répondre aux besoins de la famille autochtone contemporaine. 


\section{Résumé}

Depuis trois ans, une équipe multidisciplinaire s'est engagée à réfléchir et à développer des stratégies sur la prévention du suicide en milieu autochtone dans le cadre d'un vaste programme de recherche. Une telle entreprise pose des défis inusités à toutes les étapes de son développement. Dans cet article, l'équipe de recherche indique les difficultés rencontrées et les pistes de solution élaborées. La première partie décrit la dimension épidémiologique du suicide en milieu autochtone et elle présente les premiers résultats. La deuxième partie décrit le volet prévention et fait un bilan des essais entrepris. La troisième partie résume une recherche auprès des familles visant à mieux comprendre le contexte de socialisation dans trois milieux contrastés. Suit une brève description de l'objectif de formation de jeunes chercheurs autochtones. La discussion porte sur la capacité à répondre aux besoins de la famille autochtone contemporaine.

Mots clés: suicide - autochtone famille-socialisation - prévention.

\section{Abstract}

For the last three years, a cross-disciplinary team has been involved in developing strategies for preventing suicide in aboriginal communities with the help of a large research program. Such an initiative brings unexpected challenges at all stages of the process. In this article, our research team describes the problems met and some ideas for solutions. The first part presents the epidemiological aspect of aboriginal suicide and some preliminary results. The second part describes the prevention goals and assesses the interventions made until now. The third part summarizes a research with families where the aim is to understand the context of child-rearing in three different settings. Follows a brief overview on the training of young aboriginal researchers. The discussion concludes on the possibility to meet the needs of the contemporary aboriginal family.

Keywords: suicide - aboriginal family - socialisation - prevention.

\section{Comprendre et agir sur le suicide chez les Premières Nations Quelques lunes après I'initiation}

\section{Michel Tousignant, \\ membre chercheur, CRISE, \\ Université du Québec à Montréal.}

Arlene Laliberté,

North Queensland Health Equalities Promotion Unit, Université de Queensland, Australie.

\section{Gilles Bibeau,}

Département d'anthropologie, Université de Montréal.

\section{Denise Noël,}

Département d'anthropologie, Université de Montréal.

\section{VOLET ÉPIDÉMIOLOGIQUE}

\section{L'ÉTENDUE DU SUICIDE}

\section{EN MILIEU AUTOCHTONE}

Le suicide chez les autochtones du Canada, les Amérindiens, touche davantage les peuples les plus au nord et les plus récemment en contact avec le monde occidental. Son taux est particulièrement élevé chez les jeunes de moins de 30 ans. Ainsi, la moyenne d'âge d'un décès par suicide est de 17 ans dans la région des Terres-Cries-de-la-Baie-James du Québec en comparaison de 43 ans pour l'ensemble de cette province (Ross et François, 2007). Entre 15 et 25 ans, le taux de suicide est cinq fois plus élevé chez les autochtones que chez les non-autochtones en Colombie-Britannique entre 1987 et 1992 et cette observation s'étend à l'ensemble du Canada (Commission royale sur les peuples autochtones, 1994). Aux ÉtatsUnis, le taux de suicide des autochtones de 19 ans et moins en 1997-1998 est de 9,1/100 000 comparativement à un taux de 2,9/100 000 pour les Américains d'origine caucasienne (Centers for Disease Control and Prevention, 2003). À cela, il faut ajouter que l'incidence des morts accidentelles est d'ampleur similaire à celle du suicide chez les jeunes autochtones du Canada (Trovato, 2000).

Les taux sont très variables d'une région à l'autre et au sein même des regroupements linguistiques. Ainsi, en ColombieBritannique, 8 des 29 communautés ou bandes ont des taux de suicide nuls ou minimes, tandis que dix présentent des taux de niveau supérieur à 100/100 000, soit environ sept fois le taux de la population canadienne (Chandler et al., 2003; Westlake et May, 1986, Petawabano et al., 1994). Au Québec, le ratio de suicide hommes/femmes est proportionnellement plus élevé en milieu autochtone qu'ailleurs. Ainsi, les Inuits du Nunavik présentent un ratio hommes/femmes de 5 pour 1 (Boothroyd et al., 2001) et les adultes atikamek en Haute-Mauricie un ratio de 8 pour 1 (Laliberté, 2005). Mais comme c'est le cas presque universellement, les femmes de milieu autochtone comptent plus de tentatives de suicide que les hommes (Sigurson et al., 1994). 


\section{BRISER LES RÉTICENCES}

Une communauté ne reçoit pas d'emblée favorablement l'idée d'analyser le suicide dans son milieu à cause du danger de stigmatisation sociale. Il y a à peine 25 ans, le fait de discuter du thème du suicide et de mener des études sur les comportements suicidaires en milieu scolaire suscitait encore beaucoup de craintes au Québec. Tout le monde était d'accord pour prévenir mais moins sur la question d'analyser le phénomène en vue de mieux le connaître. Heureusement, les directions d'école et de cégep, ainsi que les comités de parents, ont compris l'urgence d'agir et les études ont permis de mieux circonscrire certains facteurs de risque associés aux idéations et conduites suicidaires chez les jeunes (Tousignant et al., 1988; 1990 ; Bastien et al., 1996; Hanigan et al., 1986). La même réserve s'est manifestée dans le cadre de la première enquête Santé Québec mais le tabou a été vaincu et le Québec a mené l'une des premières enquêtes populationnelles au plan international qui intégrait des questions sur les comportements suicidaires.

On peut donc comprendre que les Premières Nations du Québec aient eu des craintes à l'égard des études sur le suicide à la fin des années 1990. C'était l'époque où l'on observait la montée des taux de suicide et des tentatives de suicide dans plusieurs villages des Premières Nations du Nord du Québec. Quelques projets de prévention fort intéressants avaient été mis sur pied, dont le projet Migun en territoire atikamek qui consistait à créer des activités d'intégration des jeunes à la vie sociale.

En ce qui concerne la médiatisation du phénomène du suicide en milieu autochtone, un événement en 1993 a marqué un point tournant. Une série de reportages a attiré l'attention sur plusieurs accidents reliés à l'inhalation de gazoline chez des enfants du primaire à Davis Inlet, un village innu du Labrador (DeMont, 1994). Cette publication a conduit à une enquête publique mettant en lumière comment ce peuple a été repoussé loin de ses territoires traditionnels, perdant ainsi ses moyens de subsistance et sa raison d'être. Quelques années plus tard, les autorités fédérales ont décidé de déménager à nouveau le village dans un milieu plus propice à la vie traditionnelle.

\section{UNE ÉTUDE PIONNIÈRE}

La première étude ayant utilisé l'approche de l'autopsie psychologique en milieu autochtone a été menée par A. Laliberté (2005), d'origine algonquineanishnabe, dans le cadre d'une recherche de doctorat. Cette approche consiste à rencontrer les proches d'une personne décédée par suicide, afin de mieux éclairer les motifs et les circonstances du décès. L'étude s'est spécifiquement intéressée aux événements et difficultés de vie durant l'année précédant le suicide, ainsi qu'aux relations du décédé avec ses parents au cours de son enfance et de son adolescence.

Le principal obstacle rencontré dans cette recherche a été de trouver des interlocuteurs en mesure d'orienter la chercheuse vers les familles ayant vécu un décès par suicide. Les intervenants en santé mentale sont à cet égard les personnes les plus motivées et compétentes pour ce faire. Malheureusement, l'attrition du personnel en milieu autochtone est élevée et les démarches doivent être reprises au point de départ chaque fois qu'une nouvelle personne commence un contrat.

COMME DANS TOUTES

LES ENTREVUES DE TYPE

AUTOPSIE PSYCHOLOGIQUE,

MAIS ENCORE PLUS DANS

LE CONTEXTE AUTOCHTONE,

LA PRÉSENCE DE TRAUMAS

PSYCHOLOGIQUES NOMBREUX

VÉCUS DANS LES FAMILLES EXIGE

UNE CERTAINE PRUDENCE.

L'avantage d'un milieu plus isolé, en contrepartie, est qu'une fois la dynamique de la collaboration mise en place, le rythme des entrevues s'opère à une bonne cadence. Ce sont les intervenants qui approchent les membres de la famille pour présenter la recherche et c'est généralement un membre de la fratrie qui agit comme informateur.

Les entrevues posent des difficultés propres au contexte autochtone. Tout d'abord, les informateurs se soumettent à un entretien en français, une langue seconde dont ils ne possèdent pas toutes les nuances. De plus, ils se sentent mal dans une situation d'entrevue semi-structurée qui ne correspond pas au cadre de leurs échanges quotidiens. La conversation avec des étrangers prend souvent la forme du récit d'épisodes de vie racontés spontanément lorsque les circonstances s'y prêtent. Les informateurs ont parfois de la difficulté à traduire les notions plus abstraites qui touchent aux relations humaines. Par ailleurs, l'entrevue exige de préciser des dates pour situer l'enchaînement des faits avant le décès, particulièrement l'expérience d'événements difficiles comme des détails sur une relation amoureuse qui s'est rompue. Comme les peuples chasseurs possèdent une notion du temps qui ne se découpe pas nécessairement en fonction de périodes bien définies, la datation pose difficulté.

Comme dans toutes les entrevues de type autopsie psychologique, mais encore plus dans le contexte autochtone, la présence de nombreux traumas psychologiques vécus dans les familles exige une certaine prudence. Les informateurs sont évidemment affectés par le suicide d'un frère ou d'un parent, mais ils portent aussi les souvenirs troublants de leur vie de famille que nous leur demandons de partager avec nous. Et, dans ce contexte très particulier où $40 \%$ des hommes décédés par suicide possèdent un membre de leur fratrie mort de la même façon, l'entrevue peut porter sur de multiples suicides au sein d'une même famille. Par ailleurs, on observe que les familles qui participent à ce type d'étude en retirent le plus souvent des bénéfices. L'occasion de ces entrevues permet de faire un peu plus de sens autour des décès en réfléchissant sur les situations de vie qui ont produit une grande souffrance chez la personne décédée. Les règles éthiques de la recherche demandent donc d'agir avec prudence, d'être sensible à toute manifestation voilée d'angoisse suscitée par la réminiscence et de pouvoir procurer aux personnes dans le besoin le soutien psychologique nécessaire.

La personne qui mène les entrevues n'est pas non plus immunisée contre l'angoisse suscitée par les récits et elle partage le poids de la souffrance exprimée par les informateurs. Il y a nécessité de trouver sur place, dans le village, une personne de confiance avec qui partager ses états d'âme, en l'absence de toute personne proche de son réseau personnel ou de son équipe de recherche. Comme les déplacements sont coûteux et que les villages sont éloignés, on tente d'optimiser le temps passé sur place et de concentrer les entrevues, allant jusqu'à réaliser trois entrevues durant une même journée. Le soir venu, il faut dormir seul, dans un lieu non familier, avec des récits de drames qui continuent de nous habiter.

DIFFUSION ET TRANSFERT DES RÉSULTATS

Le transfert des résultats aux communautés autochtones participantes de même que leur diffusion auprès d'un plus large public exigent un certain nombre de négociations. Tout d'abord, l'étude ellemême est dès le départ encadrée par un protocole écrit avec le conseil de bande 
ou d'autres personnes concernées. On sait d'avance que ces informations mettent en relief les aspects les plus sombres de la vie quotidienne des Premières Nations, exposant ainsi leurs vulnérabilités. La diffusion des résultats se fait donc en priorité auprès des personnes impliquées dans les affaires politiques et de santé publique des communautés participantes.

La publication dans les revues scientifiques doit respecter les susceptibilités naturelles des groupes impliqués, sauvegarder l'anonymat des communautés concernées de même que mettre le suicide en contexte. La diffusion des résultats est cependant nécessaire pour faire profiter les membres des Premières Nations du Canada des leçons apprises, et certaines conclusions peuvent aussi s'appliquer aux autochtones de l'Amazonie, de l'Australie et de l'aire du Pacifique qui partagent les mêmes préoccupations.

spécifiques. L'étude par autopsie psychologique a permis de valider certaines évidences et de nuancer ou d'infirmer certaines idées reçues. Que le suicide soit étroitement associé à l'abus d'alcool est déjà connu, et cela est aussi vrai chez les hommes adultes au Québec. Mais le fait d'illustrer que, dans beaucoup de suicides, la personne décédée était intoxiquée au moment de vivre le drame qui a provoqué son suicide et, quelques heures ou quelques jours après, au moment de poser son dernier geste démontre qu'une intervention rapide auprès de personnes en grande détresse aurait pu prévenir quelques décès. On a noté également plusieurs suicides survenus en prison ou en centre de détention, ce qui exige une vigilance accrue et un soutien à ces personnes si elles sont coupées des contacts avec leurs proches. Par ailleurs, les jeunes dont la réputation sociale est fortement atteinte ont peu de recours pour

\section{L'UN DES PROBLÈMES DES ÉTUDES EMPIRIQUES DE CAS INDIVIDUELS}

\section{EST QU'ELLES NE PEUVENT PRENDRE EN CONSIDÉRATION}

\section{LES FACTEURS HISTORIQUES ET INTERCULTURELS QUI ONT CONDUIT}

\section{AUX DRAMES SOCIAUX VÉCUS PAR LES PREMIÈRES NATIONS}

\section{DANS LE PASSÉ ET DURANT LA PÉRIODE CONTEMPORAINE.}

Le présent projet doit aussi répondre à la mission de l'application des connaissances des Instituts de recherche en santé du Canada, un principe bien accueilli par les Premières Nations qui déplorent que la recherche anthropologique n'ait pas contribué par le passé à leur développement social. S'il s'avère nécessaire de pointer du doigt les injustices répétées dont ont été victimes ces peuples et qui ont aujourd'hui des répercussions sur l'équilibre socioaffectif de leurs familles, il y a danger également qu'un discours unilatéralement axé sur ce lourd héritage historique empêche les communautés de se prendre en main en se repliant dans un discours de victimisation. Il y a conséquemment un équilibre à rechercher entre reconnaître, d'une part, la souffrance et l'injustice et, d'autre part, faire appel à un sens de responsabilité communautaire empreint de courage et de détermination.

\section{SPÉCIFICITÉ DU SUICIDE} EN MILIEU AUTOCHTONE

Les observations empiriques ne permettent pas jusqu'ici de proposer un modèle général du suicide en milieu autochtone mais elles identifient certains caractères sortir de leur milieu afin d'échapper à la stigmatisation. La recherche de Laliberté a aussi permis d'identifier différentes trajectoires de vie menant au suicide. Il y a évidemment des traits communs, en l'occurrence la forte consommation, les drames personnels, et le désespoir qui les accompagne. Mais au-delà de ces constats, on voit surgir trois sous-groupes distincts: ceux qui sont victimes d'un rejet récent qu'ils ne peuvent surmonter, ceux qui sont emprisonnés et qui retournent leur frustration contre eux-mêmes et ceux dont la vie glisse inexorablement vers un décrochage d'avec tous les ancrages qui peuvent structurer leur vie.

L'un des problèmes des études empiriques de cas individuels est qu'elles ne peuvent prendre en considération les facteurs historiques et interculturels qui ont conduit aux drames sociaux vécus par les Premières Nations dans le passé et durant la période contemporaine. En faisant du cas par cas avec une minutie de détails soulignant les conflits interpersonnels de la vie en réserve, on risque d'occulter la genèse historique des problèmes contemporains, notamment l'intervention de l'État et de l'Église. Plusieurs auteurs, dont Chandler et son équipe (2003) en Colombie-Britannique, croient que les suicides sont le résultat de fractures qui ont marqué les familles au moment de la sédentarisation forcée par le gouvernement canadien et qu'ils s'inscrivent dans un contexte sociopolitique qui visait l'extinction d'une culture, entreprise coloniale aussi connue sous le nom d'ethnocide. Les principales séquelles ont été l'affaiblissement de la légitimité de la culture autochtone et la substitution des institutions à l'autorité parentale.

L'approche épidémiologique peut rapidement identifier certains facteurs de risque, tels que le surpeuplement des maisons, le manque d'emplois et le désœuvrement, la pauvreté et la difficile intégration des jeunes au monde des adultes. Mais s'ils augmentent la précarité économique et psychologique, ces facteurs ne mènent pas nécessairement au suicide. Les chercheurs ont besoin d'une collaboration étroite avec les membres des communautés autochtones pour bien saisir le contexte de l'augmentation des suicides, plus précisément après 1990. Selon nos interlocuteurs, une analyse complète exige de regarder du côté de la génération qui a précédé la cohorte récente de suicides. L'une des hypothèses explicatives repose sur le fait qu'une proportion significative des parents a vécu plusieurs années dans des écoles résidentielles ou pensionnats éloignés, avec comme conséquence de multiples traumas causés par la séparation forcée d'avec les parents et par les abus aux mains des éducateurs et des élèves plus âgés. Pour ne donner qu'un exemple du climat social à l'époque de l'acculturation forcée, la Gendarmerie royale du Canada poursuivait en forêt des enfants que leurs parents tentaient de cacher pour les soustraire aux pensionnats.

L'ère des pensionnats a donc été un point tournant dans le devenir des peuples autochtones du Canada. Plusieurs enfants, aujourd'hui parents et grands-parents, $\mathrm{y}$ ont perdu leur langue, leur culture et, fait crucial dans leur développement personnel, le lien d'attachement parental. Les autres enfants qui n'ont pas été au pensionnat ont pour leur part été sédentarisés dans des réserves. Certaines de ces réserves ont été constituées sur des sites parfois millénaires. D'autres ont été construites de toutes pièces dans de nouveaux espaces à la marge des territoires traditionnels à cause de la transformation de la topographie maritime. Ces dernières peuvent rassembler des groupes de diverses origines, anglophones et francophones, et qui, de longue date, ont vécu des relations conflictuelles. Elles offrent l'aspect extérieur d'un camp de réfugiés permanent et l'État est 
propriétaire légal d'une majorité des habitations. Il faut souligner cependant que les maisons les plus récentes sont fort convenables et s'intégreraient à n'importe quelle nouvelle banlieue québécoise.

\section{VOLET PRÉVENTION}

\section{NAISSANCE}

\section{DU PROJET WASEYA-WASENA}

À l'été 2005, notre équipe a entrepris un projet de recherche axé sur le thème de la prévention du suicide et la famille, financé par les Instituts de recherche en santé du Canada. Elle se compose des chercheurs suivants: Gilles Bibeau (anthropologie), Liesette Brunson et Michel Tousignant (psychologie), Louise Bujold et John Cutcliffe (sciences infirmières) et Duncan Pedersen (santé publique et études transculturelles). Comme le calendrier de l'appel d'offres était serré, il n'a pas permis de consulter nos partenaires des Premières Nations pour élaborer les détails du programme scientifique. Les collaborations antérieures assuraient cependant que notre plan avait été en grande partie inspiré par les réflexions et les échanges des dix années antérieures avec le milieu atikamek. Il y avait en outre le danger qu'un processus de consultation suscite des attentes frustrées en cas d'absence de subvention. De fait, les contacts réguliers avec les leaders et les aînés du milieu depuis 1996 avaient validé la priorité du thème de la famille. On avait mentionné en milieu atikamek qu'il était important de dresser une typologie des familles afin d'identifier lesquelles étaient les plus à risque.

L'un des enjeux d'un plan de recherche de cette nature est de préciser les balises générales du plan d'investigation tout en offrant une marge de manœuvre suffisante pour profiter d'opportunités qu'il ne serait pas possible de prévoir cinq années à l'avance. Au cours des mois précédant l'amorce de la présente recherche, deux rencontres nord-américaines ont permis d'établir un dialogue entre chercheurs et leaders autochtones comprenant plusieurs femmes et jeunes adultes. La première a été organisée à Montréal par Santé Canada dans un contexte pancanadien, et l'autre, à Albuquerque, a mis en présence des participants du Canada, des États-Unis et des îles du Pacifique. La première réunion a permis d'établir que les Premières Nations et les Inuits du Canada veulent participer à toutes les étapes de la recherche afin de pouvoir l'influencer et profiter de ses fruits. Cela est non seulement légitime mais aussi avantageux pour la recherche. Plus important encore est le facteur humain, le lien de confiance entre les chercheurs et les communautés, la sauvegarde de la fierté culturelle après les tentatives de destruction. La deuxième réunion au NouveauMexique a permis de constater la position privilégiée des chercheurs canadiens au regard de leurs homologues américains. Ceux-ci sont tenus par leurs organismes de subvention de la recherche en santé de présenter des devis de nature quantitative, ce qui élimine d'emblée une approche plus culturelle basée sur l'analyse du discours.

\section{PROMOTION AUPRĖS DES ENFANTS: NOKITAN II}

Cette subvention a été une occasion privilégiée de relancer l'opération Nokitan II en milieu atikamek, un projet initié par Gilles Bibeau et une équipe multidisciplinaire en vue de faire de la prévention primaire dans les écoles. Nokitan II proposait à l'origine d'intervenir en milieu éducatif pour mieux préparer les élèves à faire face à leurs difficultés socioaffectives ainsi qu'à développer des habiletés personnelles qui permettent de considérer toutes les options face à un problème. Cette initiative impliquait les autorités scolaires du village de Wemotaci, situé à une centaine de kilomètres au nord-est de La Tuque et un groupe de femmes rassemblées autour de Gaëtane Petitquay. L'intention de départ était d'adapter une expérience mise au point en milieu scolaire à Montréal avec des enfants réfugiés ayant vécu de multiples traumas (Rousseau et al., 1999).

Faire de la prévention primaire dans un milieu où la majorité des enfants présentent des vulnérabilités psychologiques oblige à prendre certaines précautions et à adapter l'intervention au plan culturel. En effet, il n'est pas possible d'aborder des thèmes comme les émotions négatives, la mort, le regard de l'autre, sans être confronté aux traumas vécus par plusieurs enfants autochtones qui ont vu ou connu une personne qui s'est suicidée ou une personne victime de violence, ce qui est moins souvent le cas pour les jeunes d'un milieu plus protégé. L'équipe a donc opté pour une approche basée sur l'art et la création pour les plus jeunes et sur la philosophie pour enfants pour les plus vieux. Cette dernière approche a été élaborée à l'Université Laval.

Introduire la philosophie pour enfants en milieu autochtone pouvait apparaître contre-intuitif au départ, en prenant en considération que la parole n'est pas un médium facile à utiliser dans ce milieu. Il faut préciser qu'en milieu atikamek, les enfants sont scolarisés dans leur langue maternelle pour les quatre premières années du primaire. À notre grande surprise, la proposition fut reçue non seulement positivement mais avec enthou- siasme. Cependant, le matériel de base utilisé dans ces programmes de philosophie pour enfants était un corpus de contes universels, certes compréhensibles pour les jeunes autochtones mais ne faisant pas partie de leur culture. L'équipe mixte de chercheurs et d'intervenants autochtones a donc décidé d'adapter un corpus de contes tirés de la culture atikamek. De fait, il fallait aussi construire ces contes qui n'étaient pas encore archivés dans une forme écrite. Un groupe de travail intégrant des femmes autochtones œuvrant dans le monde de l'éducation s'est donc donné pour tâche de rédiger une série de contes et de les adapter au contexte pédagogique de la philosophie pour enfants. Le travail est en phase finale et a constitué un défi plus grand que prévu. Les premiers contes doivent être bientôt édités, ce qui a donné lieu à un débat au sujet des droits d'auteur, puisqu'il faut à la fois reconnaître que les contes appartiennent à la culture, tout en rendant crédit aux femmes autochtones qui ont contribué à l'écriture.

La deuxième question était de savoir qui appliquerait ce programme désormais intégré au curriculum régulier. La décision fut de sélectionner en priorité des femmes autochtones plutôt que des institutrices de l'extérieur affectées aux deux écoles de Wemotaci. Tout d'abord, il y a plus de probabilités que le personnel autochtone demeure dans le village au fil des ans contrairement aux institutrices venues de l'extérieur. L'autre avantage de choisir des femmes autochtones, et cela nous le savions déjà de programmes similaires antérieurs, concerne le fait que les adultes qui procurent l'enseignement sont les premiers en quelque sorte à profiter des fruits du programme. Dans leur formation et dans le processus d'implantation, les femmes participantes sont appelées à confronter leurs problèmes personnels, à négocier avec leur entourage et à gérer leurs émotions. Elles apprennent aussi à développer des habiletés de leadership dans leur milieu, ce qui est un atout considérable dans l'optique d'une stratégie de prévention globale du suicide.

\section{L'ENQUÊTE AUPRÈS DES FAMILLES}

Comme nous l'avons déjà mentionné, un aspect important de ce programme de recherche concerne le bien-être des familles. Autant les communautés des Premières Nations que les chercheurs sont sensibilisés au fait que les familles sont le lieu des premiers attachements et qu'elles ont pour fonction d'assurer la sécurité physique et affective des enfants en même temps que de les préparer à la vie adulte. Un milieu qui ne peut offrir de garanties minimales de protection mettra 
donc l'enfant à risque de développer des troubles affectifs et contribuera à le rendre plus vulnérable au suicide dans la mesure où un certain nombre de facteurs additionnels se conjuguent. En corollaire, la famille est au centre de forces sociales qui agissent sur son équilibre et sa résilience: la religion et la spiritualité, l'identité culturelle, le réseau de parenté, l'ambiance sociale et politique, l'école, le logement et l'emploi, les modèles de vie, pour ne nommer que ces facteurs, conditionnent la capacité des parents à socialiser leurs enfants.

L'enquête sur les familles vise à comparer comment les parents se débrouillent avec leurs enfants dans trois milieux différents où nous comptons compléter cinquante entrevues dans chacun. Le premier milieu est en réserve et il inclut un village anishnabe-algonquin de l'Abitibi; le second couvre la région métropolitaine de Montréal et inclut des adultes avec enfant qui ont récemment migré en ville; le troisième comprend les régions semi-urbaines de La Tuque, Val-d'Or et Joliette.

Il n'était pas facile au point de départ de circonscrire à quoi correspondait la notion de famille en milieu autochtone. Il y a comme partout ailleurs des familles «ordinaires » avec papa-maman-enfants, mais bien d'autres modes d'arrangements qui diffèrent de ceux observés au Québec. Il y a les familles avec un seul parent, souvent une mère adolescente qui vit avec l'un ou les deux grands-parents de son enfant; les familles reconstituées avec un partenaire stable, ou installé de façon provisoire et avec un ou deux autres adultes partageant le même foyer; les familles avec enfant adopté ou servant de famille d'accueil pour des placements.

Les règles de la méthode sociologique exigent de préférence une enquête par échantillon au hasard et stratifiée. Ce type d'échantillonnage n'est pas facile à appliquer dans une réserve parce que les gens se méfient et ne savent pas très bien à quoi vont servir les informations ni même si elles ne se retourneront pas contre elles un jour. Nous avons donc décidé d'approcher les familles à travers des contacts personnels et en mettant en valeur l'objectif de trouver des solutions pour mieux assurer le bienêtre et la protection des enfants. Erreur, car tout ce qui touche au bien-être et à la protection des enfants est associé aux services de bien-être social et aux centres jeunesse, services qui ont parfois l'ingrate mission de venir enlever vos enfants quand ils croient que ceux-ci sont négligés ou qu'il y a abus au sens de la loi. Un autre obstacle est que les familles ne parlent pas facilement de leur situation à d'autres personnes de leur communauté par crainte que leurs secrets ne soient divulgués. Dans les cas où elles acceptent volontiers de le faire, il n'est pas toujours facile de s'en tenir au schéma d'entrevue. Les répondants préfèrent se raconter sous la forme d'un récit, expliquant d'où ils viennent et décrivant parfois longuement les traumas répétés qu'ils ont traversés et qui affectent encore leur rôle de parent.

\section{LE SUICIDE EST ÉGALEMENT}

\section{UN CONDENSÉ MÉTAPHORIQUE}

\section{DE CE QUI MENACE LA CULTURE}

DES PREMIÈRES NATIONS,

DE L'INTÉRIEUR COMME

DE L'EXTÉRIEUR, ET QUI

S'ENTREMÊLE À D'AUTRES

MAUX SOCIAUX COMME

\section{L'ALCOOLISME ET LA VIOLENCE.}

\section{AUTRES ACTIVITÉS COMPLÉMENTAIRES}

Un comité aviseur guide les démarches qui se font dans un village anishnabealgonquin de l'Abitibi. Ce comité sert de référence pour discuter des priorités, pour conseiller sur les approches méthodologiques qui respecteront le cadre de vie des informateurs, comme source d'information sur les événements du village, sur les crises courantes et les solutions mises en place. Ce sont des personnes qui ont des antennes dans la communauté et qui servent de point de jonction entre notre équipe et la population. Des travaux avec ce comité a surgi, en janvier 2009, un comité d'action pour faire face aux situations de crise sociale répétées et pour coordonner les divers secteurs de la vie publique: conseil de bande, sécurité publique et santé. L'un des buts est de mettre à la disposition de la population des aidants naturels dans les moments difficiles que traverse la communauté.

Deux membres de la communauté, Alex Cheezoo et Élizabeth Papatee, ont pris l'initiative d'organiser en 2008 un camp en forêt de quelques jours avec un groupe d'aînés, afin que ce milieu les aide à retrouver un peu leur identité et qu'il soit facilitateur de parole. Il faut rappeler que les Anishnabe-Algonquins vivaient sur leur territoire de pêche et de chasse jusqu'à récemment et que l'identité familiale était étroitement liée à un territoire de chasse. Nous avons donc profité de cette retraite en forêt organisée pour accompagner un groupe d'aînés. Après presque deux jours de silence, les participants se sont mis à verbaliser leurs préoccupations et nous avons pu être sensibilisés à leurs difficultés à s'intégrer à la vie familiale et à la vie communautaire.

À l'automne 2007, nous avons également eu une expérience similaire qui pourrait entrer dans la catégorie de la rechercheaction. Un intervenant auprès des jeunes de Manawan, Ulrich Ottawa, voulait organiser un camp pour les jeunes, afin de leur apprendre les techniques de survie en forêt. Comme il n'a pu obtenir de subvention, un étudiant de notre équipe, Alain Janelle (2008), a fait pression auprès des instances du gouvernement fédéral pour obtenir une subvention qui permettrait d'organiser ce camp. Son projet était de faire l'évaluation des acquis obtenus par les jeunes durant un tel camp. Le succès de la démarche a permis la tenue du camp et Alain a pu vivre deux semaines très intenses avec ces jeunes pour mieux saisir leurs états d'âme, leur façon de voir le monde et de se percevoir eux-mêmes. Ce camp a démontré de façon concluante que les jeunes sont prêts à participer à des activités qui leur donnent des responsabilités.

Comme mentionné plus haut, il existe un taux élevé de placements et de signalements en milieu autochtone, ce qui oblige légalement les services sociaux à intervenir. À cet égard, l'un des objectifs de l'équipe est de réfléchir sur les façons de prévenir les placements d'enfants parce que l'ampleur du phénomène est en train de reproduire des séquelles similaires à celles laissées par les pensionnats dans la jeune génération actuelle. Le principal défi est de prévenir les placements ou de préserver le lien d'attachement du parent avec l'enfant s'il y a placement. Une visite de trois membres de l'équipe dans un centre de traitement pour enfants autochtones souffrant de malnutrition dans l'État de Mato Grosso do Sul (Brésil) en octobre 2007 nous a sensibilisés aux effets pervers des placements. Dans cet hôpital agréable, propre, doté d'un personnel compétent, les enfants retrouvaient rapidement leur santé. Cependant, les parents pendant ce temps perdaient le peu d'attachement qui avait en partie été à la source de la malnutrition et ils avaient de la difficulté à reprendre contact avec leurs enfants dont ils s'étaient davantage éloignés pendant leur absence du foyer.

\section{LA FORMATION}

DE CHERCHEURS AUTOCHTONES

Une des contributions importantes de ce type de recherches est la formation d'étudiants universitaires ou de professionnels 
autochtones. En ce qui concerne le milieu académique, notre programme de recherche a aidé à l'achèvement d'une thèse de doctorat et à la mise en marche d'une deuxième par Nibisha Sioui, une étudiante d'origine huronne ayant passé son enfance en milieu anishnabe-algonquin, qui s'intéresse à la résilience des familles. Arlene Laliberté a été la première coordinatrice du projet et elle est maintenant en stage postdoctoral à l'Université du Queensland en Australie où elle poursuit un travail similaire en prévention du suicide. D'autres étudiants autochtones de premier et de deuxième cycle participent aussi à notre projet. Danielle Rousselot, du village innu-montagnais de Betsiamites, complète une maîtrise en éducation à l'Université de Montréal avec un mémoire qui porte sur l'évaluation de Nokitan II. Leur présence inspire confiance aux communautés, parce que notre projet n'est plus perçu comme seulement l'affaire de Blancs étrangers.

L'expérience montre qu'il n'est pas toujours facile pour des étudiantes autochtones de travailler dans leur milieu, même si ce n'est pas dans leur village d'origine. L'écoute des drames du quotidien produit chez elles une résonance émotive plus forte que chez des non-autochtones qui ne s'identifient pas autant aux situations rencontrées. Leur avancement académique contribue par ailleurs au sentiment de fierté des communautés autochtones, qui voient des modèles de réussite se développer sous leurs yeux, et les jeunes sont plus à même de réaliser que l'université est à leur portée.

Nous avons également un chercheur de terrain, diplômé en travail social, qui œuvre dans son village d'origine. Cette situation aide certainement à gagner la confiance des gens de l'intérieur et, pour nous, à mieux connaître ce qui se passe. En même temps, la recherche procure un cadre de questionnement à ce chercheur et il peut par la suite partager ses préoccupations avec d'autres leaders du village.

\section{RÉPONDONS-NOUS AUX BESOINS}

\section{DES COMMUNAUTÉS?}

En recherche, il n'est pas facile d'identifier ce que veulent les communautés et d'y répondre adéquatement. Dans notre domaine d'expertise, il est indéniable que la question du suicide est perçue comme une urgence. On ne peut voir disparaître des jeunes à chaque année et d'autres attenter sérieusement à leurs jours sans vouloir améliorer le sort de ceux qui restent et arrêter l'hémorragie. Le suicide est également un condensé métaphorique de ce qui menace la culture des Premières Nations, de l'intérieur comme de l'exté- rieur, et qui s'entremêle à d'autres maux sociaux comme l'alcoolisme et la violence. Cependant deux réflexes chez les autochtones freinent la collaboration avec la recherche académique: le besoin d'agir immédiatement et le besoin d'être autonome et de conserver sa fierté.

On nous répète que, depuis longtemps, les chercheurs viennent tirer des informations et repartent ensuite embellir leur carrière universitaire sans profit pour les gens qui ont contribué à leur savoir. La recherche est donc comme une des facettes du colonialisme. Et lorsqu'on veut mettre fin à une situation de crise, que le diagnostic paraît évident et sans besoin de le documenter davantage après des montagnes de rapports, quel est l'avantage de rassembler encore plus d'informations?

L'un des moyens de sortir de cette impasse est de réfléchir à plusieurs, à bâtons rompus et sans langue de bois, pour regarder les problèmes en face et se rendre compte que les solutions ne sautent pas immédiatement aux yeux ou ne sont pas faciles à implanter. À cet égard, nous rencontrons une façon parfois assez directe d'exprimer les choses, une authenticité surprenante, une autocritique nuancée, et également une espérance de voir des améliorations, fondée sur une spiritualité inspirée par la renaissance culturelle.

Des évidences sautent aux yeux de nos collaborateurs, et peu à peu à ceux des chercheurs, entre autres les séquelles psychologiques et identitaires de l'épisode des pensionnats, puis la plaie moderne des placements d'enfants. Entre mettre le doigt sur ces réalités et relever le défi, il y a évidemment un grand écart. C'est peut-être là que la recherche permettra de mieux éclairer des phénomènes complexes et qui correspondent à des dynamiques diversifiées et de suggérer des voies de solution adaptées aussi bien à la culture qu'à toutes les spécificités de chaque famille et de chaque communauté, car il existe en effet d'importantes variations entre chaque village.

Au départ, le rôle du chercheur est assimilé à la tâche d'un acteur qui peut se payer le loisir de rassembler l'expérience de multiples observateurs pour comprendre la vie sociale et domestique des autochtones. Ces gens sont nombreux, ouverts à la collaboration parce qu'ils ressentent un peu d'isolement, et parce qu'ils ont à cœur la prévention et l'amélioration de la qualité de vie.

L'un des objectifs est de mieux connaître la famille contemporaine et nous avons mis sur pied la démarche classique des entrevues à domicile. L'approche offre une fenêtre privilégiée, mais ce programme est extrêmement difficile à réaliser. Aussi, nous devons compléter notre portrait par la recherche dans l'action; plus précisément, profiter de toutes les activités et opportunités pour brosser un tableau plus complet. Les programmes de retraite en forêt sont des opportunités exceptionnelles où les gens se sentent libres de s'exprimer et, surtout, de témoigner en solidarité avec leur groupe d'appartenance. Des activités de partage, comme camper en nature, des repas informels, accompagner une personne dans une sortie, sont d'autres occasions de lire les situations à travers la perspective de chacun. L'important est aussi de sentir les rêves, les espoirs qui se traduisent dans le récit des drames. Sans cela, nous ne pourrions nous-mêmes tenir le coup très longtemps. À ce titre, les autochtones nous apprennent énormément parce qu'ils ont passé à travers des tragédies aux dimensions disproportionnées au regard de nos petits drames bourgeois. En même temps, nous leur apportons une ouverture sur le monde extérieur que nous représentons. Ce monde extérieur n'est pas seulement celui des Blancs, mais également celui des autres peuples autochtones, au Québec, au Canada, et à travers le monde. Nos cumulons dans l'équipe une longue expérience de travail anthropologique en Afrique, en Amérique latine et chez les Inuits.

La question du respect de l'autonomie se pose lorsque les communautés autochtones voient s'amener chez elles des visages pâles, pire, des gens de la grande ville; elle suscite des tensions évidentes, compréhensibles, mais aussi surmontables. Cela est vrai autant des villages autochtones que des milieux périphériques de la province de Québec. Tout d'abord, il y a déjà une circulation importante entre nos deux univers. Un certain nombre de Québécois, des francophones, et d'autres également qui viennent aussi loin que d'Afrique, travaillent dans les réserves. Certains y sont depuis de nombreuses années. Il y a également des touristes de passage. De même, les leaders ont une très bonne expérience de la vie québécoise: ils ont étudié dans les institutions supérieures, vécu à la ville, et ils se sont aussi intéressés à d'autres cultures des Premières Nations en Amérique du Nord. La télévision câblée est omniprésente; le village de Manawan a priorisé il y a déjà longtemps la connexion technologique au monde extérieur par la construction d'un réseau de télécommunications et il en a même fait profiter des villages francophones situés sur le parcours.

Il est indispensable que la prévention du suicide soit prise en main par les Premières Nations. Une intervention efficace contrôlée essentiellement de l'extérieur risquerait d'être contre-productive. Le défi du côté 
des chercheurs et des intervenants est de nous rendre utiles en laissant le plus d'initiative à la population locale. Nous avons réussi avec Nokitan II à donner un rôle pivot aux femmes de la communauté et elles en tirent beaucoup de fierté. D'autres villages voudraient faire leur ce projet, parce qu'ils en ont entendu parler et qu'ils y voient un excellent moyen d'améliorer le sort de leurs enfants. Mais voilà, l'appropriation a été tellement forte que la communauté est maintenant réticente à voir son projet déménager ailleurs sans négocier des «droits d'auteur».

$$
\star * *
$$

L'Institut de santé des autochtones, l'un des treize instituts composant les Instituts de recherche en santé du Canada, nous avait prévenu que la recherche dans ce milieu amènerait probablement une nouvelle conceptualisation de la nature même de la recherche. Mais comment faire de la recherche scientifique qui soit à la fois enracinée, utilisable, motivante et respectueuse?

La dichotomie entre recherche fondamentale et recherche appliquée occulte probablement une réalité de la quête de la connaissance, soit la part du patient ou de l'indigène. Freud ne serait pas le père de la psychanalyse si une patiente libérée avant son époque ne lui avait pas signifié fermement qu'il devait se taire et la laisser parler. La pharmacologie moderne a ratissé les jungles et les jardins traditionnels du monde entier pour pénétrer les secrets de la guérison de nombreuses maladies au point même d'avoir l'insolence de mettre des droits d'auteurs sur des plantes connues pour leurs vertus médicinales depuis des millénaires par la médecine ayurvédique. La science est progressivement devenue une pratique aseptisée, contrôlée, appuyée par des régiments de techniciens et d'ingénieurs, mais surtout par des multinationales pharmacologiques qui, trop souvent, manipulent la vérité à des fins de profit. Dans le verger de Newton ou dans la baignoire d'Archimède, la science fondamentale ne s'est-elle pas inspirée d'intuitions créatrices? Il n'y a donc pas de honte ou de romantisme déplacé à vouloir penser de nouveaux paradigmes de recherche qui intégreront la riche expérience de ceux et celles qui sont les observateurs de premier plan, les membres mêmes des communautés autochtones.

Le savoir sera d'autant plus facile à transmettre, et porteur de bénéfices, qu'il aura été construit en collaboration avec les gens qui l'appliqueront. Pour cela, les intervenants doivent s'initier à la recherche et les chercheurs se sensibiliser aux urgences des intervenants et des populations.

\section{Bibliographie}

BASTIEN, M.F., M. TOUSIGNANT et S. HAMEL (1996). "Étude comparative de l'intégration scolaire chez des adolescents suicidaires et non suicidaires victimes de carence parentale », Santé Mentale au Québec, $\mathrm{n}^{\circ} 21$, p. 33-52.

BOOTHROYD, L.J., L.J. KIRMAYER, S. SPRENG, M. MALUS et S. HODGINS (2001). «Completed suicides among the Inuit of Northern Quebec, 1982-1996: A case-control study ", Canadian Medical Association Journal, vol. 18, no 164-166, p. 749-755.

CENTERS FOR DISEASE CONTROL AND PREVENTION, DEPARTMENT OF HEALTH AND HUMAN SERVICES (2003). "Injury mortality among American Indian and Alaska Native children and youth - United States, 1989-1998 », Morbidity and Mortality Weekly Report, vol. 52, no 30, p. 697-701.

CHANDLER, M.J., C.E. LALONDE, B.W. SOKOL et D. HALLETT (2003). «Personal persistence, identity development, and suicide: A study of Native and non-Native North American adolescents ", Monographs of the Society for Research in Child Development, vol. $68, \mathrm{n}^{\circ} 2$, p. 1-130.

COMMISSION ROYALE SUR LES PEUPLES AUTOCHTONES (1995). Choisir la vie: un rapport spécial sur le suicide chez les autochtones, Groupe Communication Canada Édition.

DEMONT, J. (1994). "The fight of a lifetime », Macleans, janvier, p. 21-26.

HANIGAN, D., M. TOUSIGNANT, M.-F. BASTIEN et S. HAMEL (1986). «Le soutien social suite à un événement critique chez un groupe de cégépiens suicidaires ", Revue Québécoise de Psychologie, vol. 7, $\mathrm{n}^{\circ}$ 3, p. 63-81.

JANELLE, Alain (2008). «Évaluation du processus et des effets d'une activité de prévention du suicide chez les jeunes Atikamekw de la communauté de Manawan », Honours thesis comme exigence partielle dans le cadre du cours Activité de recherche en psychologie (PSY5900), Université du Québec à Montréal.

LALIBERTÉ, A. (2005). Suicide et événements de vie dans la communauté attika$m e k h$, thèse de doctorat inédite, Université du Québec à Montréal.

PETAWABANO, B.H., E. GOURDEAU, F. JOURDAIN, A. PALLISER et J. COSSETTE (1994). La santé mentale et les autochtones du Québec, Montréal, Gaëtan Morin éditeur.

ROSS, L. et H. FRANÇOIS (2007). «Profil du suicide au Québec 1981-2005 p: mise à jour en 2007 », Ministère de la Santé et des Services sociaux du Québec, en ligne, <http:// www.msss.gouv.qc.ca/sujets/prob_sante/ pdf/Profil-suicide-fev07.pdf $>$, consulté le 2009-03-23.

ROUSSEAU, C., D. BAGILISHYA, N. HEUSCH et L. LACROIX (1999). "Jouer en classe autour d'une histoire: ateliers d'expression créatrice pour les enfants immigrants exposés à la violence sociale ", P.R.I.S.M.E. Psychiatrie, recherche et intervention en santé mentale de l'enfant, $\mathrm{n}^{\circ} 28$, p. 88-103.
SIGURSON, E., D. STALEY, M. MATS, K. HILDAHL et K. SQUAIR (1994). "A five year review of youth suicide in Manitoba", Canadian Journal of Psychiatry, vol. 39, $\mathrm{n}^{\circ} 8$, p. 397-403.

TOUSIGNANT, M., S. HAMEL et M.-F. BASTIEN (1988). «Structure familiale, relations parents-enfants et conduites suicidaires à l'école secondaire», Santé Mentale au Québec, n 13, p. 79-93.

TOUSIGNANT, M., S. HAMEL et M.-F. BASTIEN (1990). "Le suicide chez les jeunes», Interface, vol. $11, \mathrm{n}^{\circ} 3$, p. 22-28.

TROVATO, F. (2000). "Canadian Indian mortality during the 1980s », Social Biology, vol. $47, \mathrm{n}^{\circ} 1-2$, p. $135-145$.

WESTLAKE, V.W.N. et P.A. MAY (1986). "Native American suicide in New-Mexico, 1957-1979: A comparative study», Human Organization, vol. 45, n 4, p. 296-309. 